\title{
In-Silico Design of Novel Glucagon-Like Peptide 1 Mutants as Candidate for New Peptide Agonist Drugs
}

\author{
Tony Sumaryada ${ }^{1,2 *}$, Ajeng Widya Roslia ${ }^{1}$, Alfi Afifah ${ }^{1,3}$, Setyanto Tri Wahyudi ${ }^{1}$, Agus Kartono ${ }^{1}$ \\ ${ }^{1}$ Computational Biophysics and Molecular Modeling Research Group (CBMoRG), Department of Physics, Bogor Agricultural \\ University, Bogor, Indonesia \\ ${ }^{2}$ Tropical Biopharmaca Research Center, Bogor Agricultural University, Bogor, Indonesia \\ ${ }^{3}$ Madania Secondary School, Telaga Kahuripan, Parung Bogor, Bogor, Indonesia
}

\section{ARTICLE INFO}

Article history:

Received November 15, 2019

Received in revised form October 3, 2020

Accepted October 10, 2020

\section{KEYWORDS:}

Free energy perturbation, glucagon-like peptide 1 interaction stability, mutation,

protein-protein docking simulation,

type 2 diabetes mellitus

\begin{abstract}
The binding of glucagon-like peptide 1 (GLP-1) incretin hormone and its receptor GLP-1R plays an important role in the human body. The GLP-1 acts as the insulin secretion stimulator through a GLP-1R agonist activation to avoid the type 2 diabetes mellitus problem. A recent development in computational sciences has enabled us to design a new GLP-1 mutant which has a better binding stability to GLP-1R. In this paper, we have conducted an in-depth analysis of protein-protein docking of GLP-1 and GLP-1R receptor to determine the responsible factors affecting the binding stability. The protein-protein binding stability was analyzed by performing the point mutations on the GLP-1 structure and running the molecular dynamics simulation of the docked structures. Five mutants, Lys20Arg, Lys20His, Lys20Ser, Lys20Gly, and Lys20Ala, has been created computationally and docked with GLP-1R and tested via a molecular dynamics simulation and the free energy perturbation calculation to search for the best-binding mutant. Our results have shown that the Lys20His mutant design has the best potential to be developed as a new peptide agonist drug based on its binding affinity and structural integrity as compared to other mutants and the peptide agonist drugs available in the market exenatide, and liraglutide.
\end{abstract}

\section{Introduction}

The type 2 diabetes mellitus (T2DM) is a type of diabetes mellitus with the highest number of patients, covering $90-95 \%$ of the total diabetes mellitus case in the world. (American Diabetes Association 2012; Olokoba et al. 2012). This chronic metabolic disease is characterized by high blood sugar levels (hyperglycemia) caused by insulin resistance (reduction of insulin secretion or insulin function) (Weyer et al. 1999). The insulin secretion in the body occurs through various mechanisms, one of them depends on the role of incretin hormone. The Incretin hormone itself is a gut hormone that can stimulate insulin secretion in the body. One of incretin hormone that has a significant role in insulin secretion is GLP-1.

Protein GLP-1 (Glucagon-like peptide 1) is an incretin hormone that mainly produced in

\footnotetext{
* Corresponding Author

E-mail Address: tsumaryada@apps.ipb.ac.id
}

enteroendocrine $\mathrm{L}$ cells of the gut shortly after eating or increasing glucose in the blood (Doyle and Egan 2007). This protein stimulates insulin secretion in the body by activating the GLP-1R agonists in pancreatic $\beta$ cells to encourage adenylyl cyclase activation and cAMP (cyclic Adenosine Mono Phosphate) generation. This cAMP production will activate PKA (Protein Kinase A) and EPAC (Exchange Proteins Activated by cAMP) that play an essential role in insulin secretion (Meloni et al. 2012). The GLP-1 performs critical functions in the body such as: stimulating the insulin secretion from the pancreatic $\beta$-cells, stimulating the biosynthesis of insulin and insulin sensitivity, increasing the $\beta$-cell proliferation and apoptosis protection, inhibiting the glucagon secretion, gastric emptying, and food intake (Drucker 2001; Li et al. 2010; Quoyer et al. 2010). Those specific functions make the GLP-1 protein as one alternative that can be applied in treating the T2DM and as primary therapy for metabolic diseases such as obesity (Ahrén and Ahren 2011; Mostafa et al. 2015; Anderberg et al. 2016). The GLP-1 protein 
lifetime in the body is only about 2 minutes before rapidly deactivated by an enzyme called DPP IV (Dipeptidyl peptidase 1V) (Baggio and Drucker 2007). Research on T2DM treatment is currently focused on increasing the activity of GLP-1R by developing GLP1 analog hormone or developing the DPP-IV inhibitor to maintain the amount of GLP-1 in the body. Some GLP-1 analogs such as exenatide and liraglutide have been found with longer-acting effects and considered more potent than GLP-1 wild-type (Briones and Bajaj 2006; Vilsbøll et al. 2008). Those analogs have similar amino acid structures with GLP-1 protein and interact with GLP-1R receptors (De Maturana et al. 2003; Russell-Jones 2009; McClean et al. 2011). The success of these two analogs in T2DM treatment provides an excellent opportunity to develop other analogs with better performance and stability.

Study on protein modifications (or protein engineering) to improve the protein function and stability continues to be a significant interest topic in drug design, biotechnology, and food industries (Bai et al. 2016). This effort is mainly focused on the optimizing the expression, purification, modification, formulation, storage and structural studies of proteins (Deller et al. 2016). For the drug design field, the binding (docking) stability of ligand to the receptor are significant especially when we try to create a new design of protein agonist drugs. The stability and functionality of proteins depend on many factors, such as the electrostatic interactions, structural flexibility and also the amino acid (residue) sequence of the proteins (Jelesarov and Karshikoff 2009). Replacing one amino acid with the other (the point mutation method) could lead to the stabilization or destabilization of the whole structure and the functionality of the proteins (Jonson and Petersen 2001; Pikkemaat et al. 2002). The computational approach on the protein-protein interaction via molecular dynamics simulations and docking method might help us in designing new protein agonist drugs which further can be tested by in vivo and in vitro (wet experiments) methods.

This paper is aimed to design and evaluate the performance of GLP-1 mutants using computational approach, the molecular dynamics simulation, and protein-protein docking simulation. The proteinprotein docking of GLP-1 ligand and GLP-1R receptor were performed to simulate the binding between those two proteins. The molecular dynamics simulation of those docked structures of GLP-1 and
GLP-1R receptor was used to identify the essential salt-bridge pairs which always occurred in the simulation and play crucial roles in maintaining the stability of the ligand-receptor binding. Once the salt-bridge couple recognized, the point mutations conducted on ligand's amino acid to design some GLP-1's mutants. The molecular dynamics simulation then performed on the mutants to find the most stable mutant which has better binding stability and the docked structure integrity. The stability interaction of this GLP-1 mutant was also compared to the available peptide agonist drugs (GLP-1 analogs) available in the market, such as exenatide, and liraglutide.

\section{Materials and Methods}

The initial structure of the protein was downloaded from protein data bank (www.rcsb.org) with the PDB code name 1D0R for GLP-1 (Chang et al. 2001), 3C59 for GLP-1R (Runge et al. 2008), 1JRJ for exenatide (Neidigh et al. 2001), and 4APD for liraglutide (Worldwide PDB 2014). The GLP-1 protein consists of 30 residues, during exenatide 39 residues, and liraglutide 31 residues. The wild-type sequence of the GLP-1 peptide is shown in Table 1, and the site chosen for mutational study is highlighted. The residue sequence of downloaded GLP-1R protein was started from 20 to 131, but to simplify the simulation and analysis, we renumbered the residue to begin from 50 to 152. The protein structures were prepared using VMD 1.9 (Visual Molecular Dynamics) program (Humphrey et al. 1996) before running the molecular dynamics simulations. Preparation of protein structure consists of removing ligands and water molecules, setting the center of coordinate, creating the topological files, creating the water box $( \pm 14 \AA$ from the edge of protein structure in the $\mathrm{x}-\mathrm{y}-\mathrm{z}$ coordinates), neutralizing the molecules by adding $\mathrm{NaCl}$ ions $(0.15 \mathrm{~mol} / \mathrm{l})$. A series of minimization process (for $10 \mathrm{~ns}$ ) then performed to put the protein

Table 1. The amino acid sequence of a GLP-1 peptide. The site chosen for mutational study is highlighted

\begin{tabular}{ll}
\hline Ligand & \multicolumn{1}{c}{ Amino acid sequence } \\
\hline GLP-1 peptide & His1-Ala2-Glu3-Gly4-Thr5-Phe6-Thr7- \\
& Ser8-Asp9-Val10-Ser11-Ser12-Tyr13- \\
& Leu14-Glu15-Gly16-Lys17-Ala18-Ala19- \\
& Lys20-Glu21-Phe22-Ile23-Ala24-Trp25- \\
& Leu26-Val27-Lys28-Gly29-Arg30 (NH2) \\
\hline
\end{tabular}


structure in the minimum energy state (the most stable conformation) before proceeding to the next step, i.e., protein-protein docking simulation.

The protein-protein docking simulations were performed between the receptor protein GLP-1R and ligand proteins (GLP-1, exenatide, and liraglutide) using an online server ZDOCK (Chen et al. 2003; Pierce et al. 2014). For each docking simulation, the Z-dock server will produce ten binding modes. Based on the Z-dock scoring function the first compulsory mode (complex.1.pdb) is taken as best docked structure (as seen in Figure 1) and will be used for molecular dynamics simulation.

The docked structures produced by Z-DOCK then simulated again using NAMD (Not Just Another Molecular Dynamics) version 2.9 software (Phillips et al. 2005) to check the structural integrity and identify
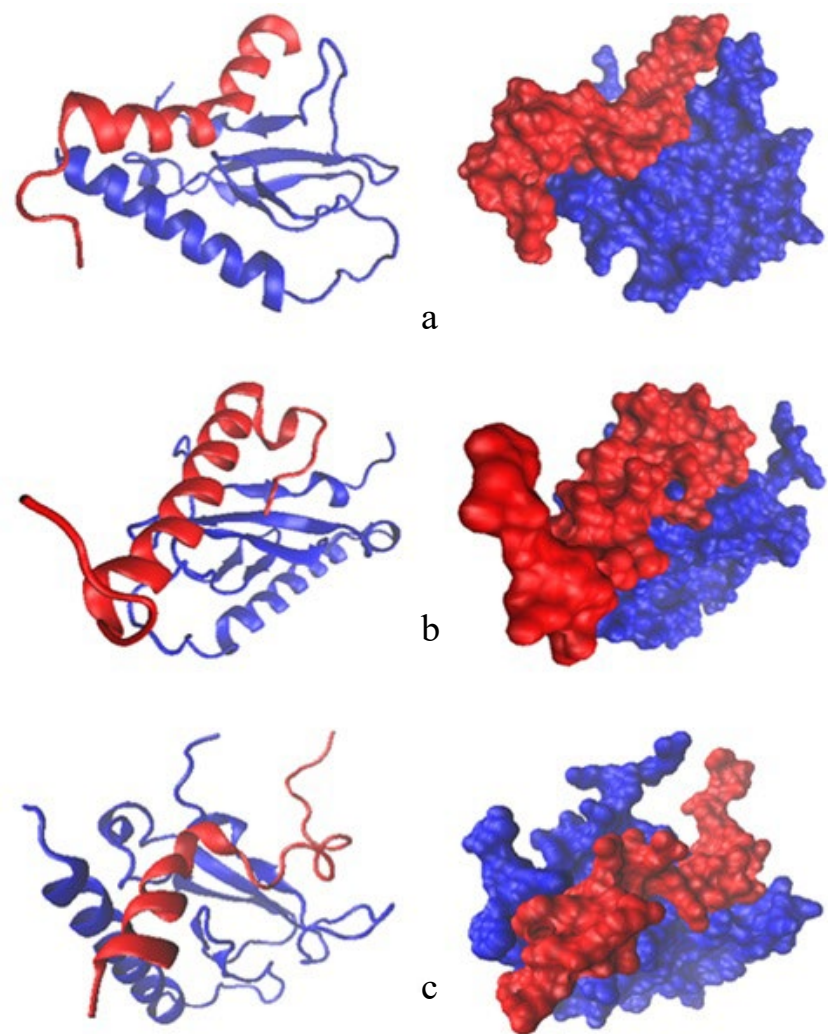

Figure 1. The docked structures of the ligand-receptor interaction produced by Z-DOCK server. The GLP$1 \mathrm{R}$ receptor docked with (a) GLP-1 (b) Exenatide, and (c) Liraglutide. The blue color represents the receptor protein, and the red color represents ligand protein. The left panels (images) are in the secondary structure representation, while the right groups (images) are in the surface representation factors responsible for the stability of ligand-receptor interactions. The molecular dynamics simulation were comprised of minimization (10 ns), heating and equilibration ( $5 \mathrm{~ns}, 310 \mathrm{~K}$ ), and the production run of molecular dynamics simulation (50 ns) with 2 fs time step and SHAKE constraint (Ryckaert et al. 1977). For the accuracy, the simulation of the docked structure of GLP-1 wild-type and GLP-1R was run three times. The first run was done with the initial structure came from the Z-Dock result (complex.1.pdb). The second run used the initial structure came from the $10 \mathrm{~ns}$ frame of the first simulation, while the third run used the initial structure came from the 20 ns frame of the first simulation. All simulations were performed using the same parameters system and conditions.

To identify the factors or residues responsible for the binding stability of the ligand-receptor structure, some parameters must be analyzed such as the RMSD (root mean square deviation), SASA (solvent accessible surface area), radius of gyration, RMSF (root mean square fluctuation), and the binding energy between ligands and receptor. Based on those results, we can determine the essential residue (or residues) that maintain the interaction stability. Since we can only mutate ligand (modify ligand) and not the receptor, so the residue that we analyze should be in ligand structure. Once the residue was identified, then we can evaluate the role of this residue by making point mutations. The free energy perturbation (FEP) approach was used to calculate the effect of mutation to the energetic stability of the docked structure by using a dual topology paradigm under aqua and vacuum (waterless condition) (Henin et al. 2017), as illustrated in Figure 2.

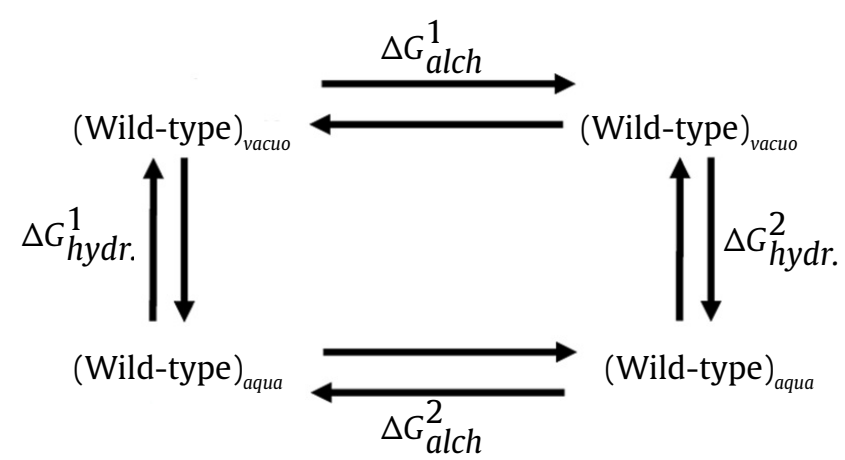

Figure 2. Thermodynamics cycle of solvent-free energy changes 
In the FEP approach, the energy of each process above was defined as:

$$
\begin{gathered}
\Delta \Delta G=\Delta G_{a l c h}^{2}-\Delta G_{a l c h}^{1}=\Delta G_{h y d r}^{2}-\Delta G_{h y d r}^{1} \\
\Delta \Delta G=\Delta G_{\text {aqua }}^{2}-\Delta G_{\text {vacuo }}^{1}
\end{gathered}
$$

where,

$\Delta G_{\text {alch }}^{1}:$ the energy required for mutations in a vacuum $(\mathrm{kcal} / \mathrm{mol})$

$\Delta G_{\text {alch }}^{2}:$ the energy required for mutations in a vacuum $(\mathrm{kcal} / \mathrm{mol})$

$\Delta G_{h y d r}^{1}$ : the energy required to dissolve the wildtype $(\mathrm{kcal} / \mathrm{mol})$ $\Delta G_{h y d r}^{2} \quad \begin{aligned} & \text { : the energy required to dissolve the mutants } \\ & \text { (kcal } / \mathrm{mol})\end{aligned}$

For the calculation of the binding energy of ligandprotein with receptor-protein, the MM/PBSA(Molecular Mechanics Poisson Boltzmann Surface Area) and MM/ GBSA(Molecular Mechanics Generalized Born Surface Area) methods within the AMBER program was used (Hou et al. 2011). This approach follows some equations below:

$$
\begin{gathered}
\Delta G_{\text {mmgbsa }}=G^{\text {complex }}-G^{\text {receptor }}-G^{\text {ligand }} \\
\Delta G_{\text {bind }}=\Delta H-T \Delta S \approx \Delta E_{M M}+\Delta G_{\text {solvation }}-T \Delta S \\
\Delta E_{M M}=\Delta E_{\text {internal }}+\Delta E_{\text {electrostatic }}-\Delta E_{v d w} \\
\Delta G_{\text {solvation }}=\Delta G_{P B / G B}+\Delta G_{S A S A}
\end{gathered}
$$

where,

$\Delta G_{M M} \quad$ : the total gas phase mechanical energy of the molecular system which includes the internal energy (energy binding, angle, and dihedral) van der Walls ( $\Delta E_{v d w}$ ) and the electrostatic interaction $\left(\Delta E_{\text {electrostatic }}\right)$ in the unit of $\mathrm{kcal} / \mathrm{mol}$

$\Delta G_{\text {solvation }}$ : the free energy of ligand bonds removal, determined from Poisson Boltzmann (PB)/ Generalized Born(GB) and SASA calculations, in the unit of $\mathrm{kcal} / \mathrm{mol}$

$-T \Delta S \quad$ : the product of temperature (at $310 \mathrm{~K}$ ) and the solubility entropy arising from the change of degrees of free molecule dissolved (kcal/ $\mathrm{mol}$ )

\section{Results}

The molecular dynamics simulation of the docked structure of the GLP-1 wild-type and the GLP-1R receptor was run three times for 50 ns each, and at $310 \mathrm{~K}$. The results, as seen in Figure 3, indicate that those three runs of simulation did not produce a drastic conformational change as shown by the relatively low value of RMSD of the backbone of protein-protein docking.

The salt-bridge interactions between GLP- 1 and GLP$1 \mathrm{R}$ were tabulated in Table 2 and become the selection basis for protein mutation. Two salt-bridges, Lys20Glu89, and Glu21-Arg142 have been identified as the most frequent salt-bridge pairs that appeared during simulations. The dynamics of those mutations in terms of electrostatic energy and the interaction distance of Lys20-Glu89 and Glu21-Arg142 mutants were shown in Figure 4. The degree of the mutation of GLP-1 can be expressed in terms of solvation free energy changes as shown in Figure 5 . Lambda $=0$ denotes that the protein structure is entirely in the wild-type form, while lambda $=1$ indicates that the protein structure is altogether in the mutant form.

The binding stability of GLP-1's mutants and GLP-1R at physiological temperature $(310 \mathrm{~K})$ in terms of some molecular dynamics structural's parameters (RMSD, Rg, SASA, and RMSF) is shown in Figure 6. The dynamics of the tertiary structures of the mutant's complexes are shown in Figure 7. In that figure, the attachment and detachment of GLP-1 and its mutants with GLP-1R during molecular dynamics simulation can be tracked to evaluate the structural integrity of the complexes. As seen in Figure 7, the best mutant that maintains the structural integrity of the ligand-receptor complex is Lys20His. The binding of GLP-1 wild-type, Lys20His mutant, exenatide, and liraglutide to GLP-1R then compared in Figure 8. The binding stability as seen in Figure 8 shows the evolution of the structural parameter of the complexes, such as RMSD, Rg, SASA, and RMSF. In terms of energetical parameters, the binding affinity

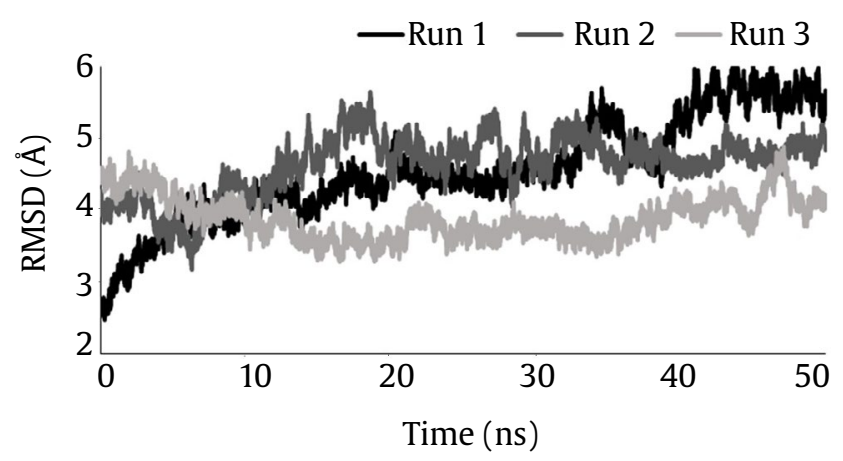

Figure 3. The RMSD value of the docked structure of GLP-1 and GLP-1R during simulation of each run 
of those complexes was compared using MM/PBSA (relative binding energy) and MM/GBSA method and tabulated in Table 3.

Table 2. Salt-bridge pairs between GLP-1 and GLP-1R receptor appears during each simulation

\begin{tabular}{lll}
\hline Run 1 & Run 2 & Run 3 \\
LYS20-GLU89 & LYS20-GLU89 & LYS20-GLU89 \\
GLU21-ARG142 & GLU21-ARG142 & GLU21-ARG142 \\
GLU15-ARG152 & & GLU15-ARG152 \\
GLU3-ARG61 & & GLU3-ARG61 \\
ASP9-ARG61 & & GLU3-ARG65 \\
& & GLU21-ARG123 \\
\hline
\end{tabular}

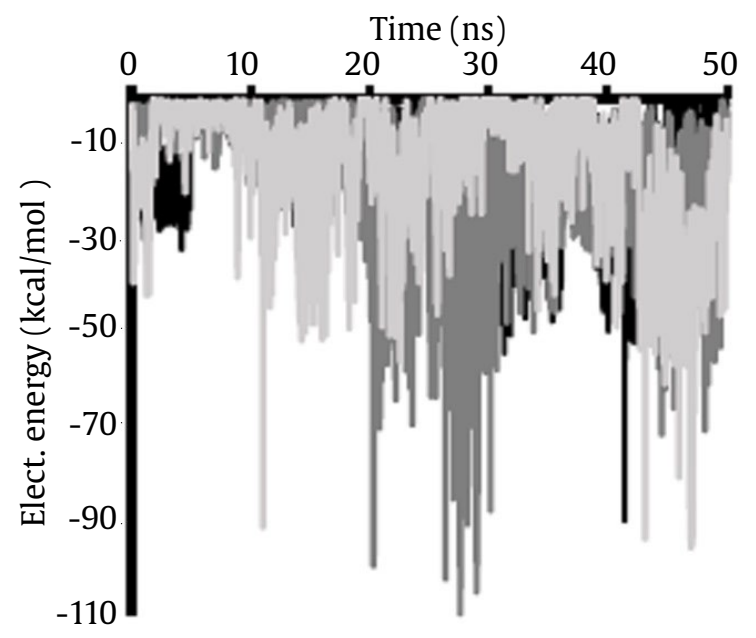

$\mathrm{a}$

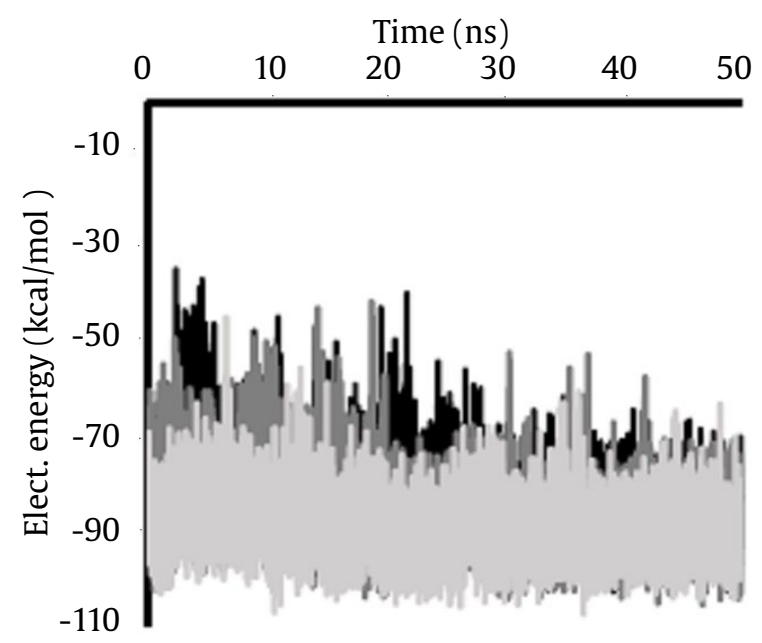

C

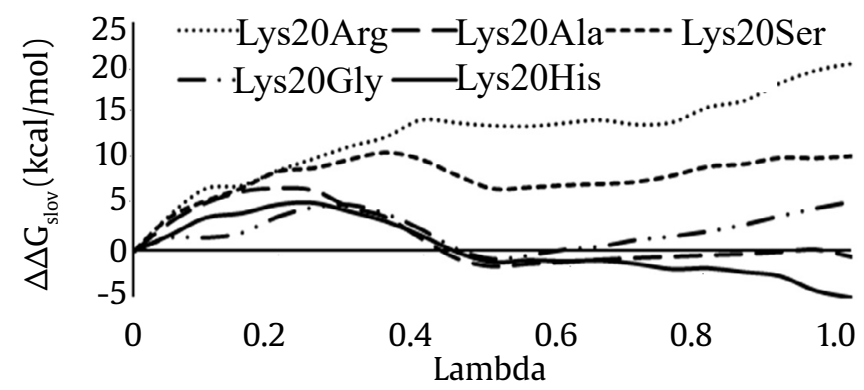

Figure 5. The changes of solvation free energy of GLP-1 mutant candidates. Lambda $=0$ denotes that the protein structure is entirely in the wild-type form, while lambda = 1 indicates that the protein structure is altogether in the mutant form

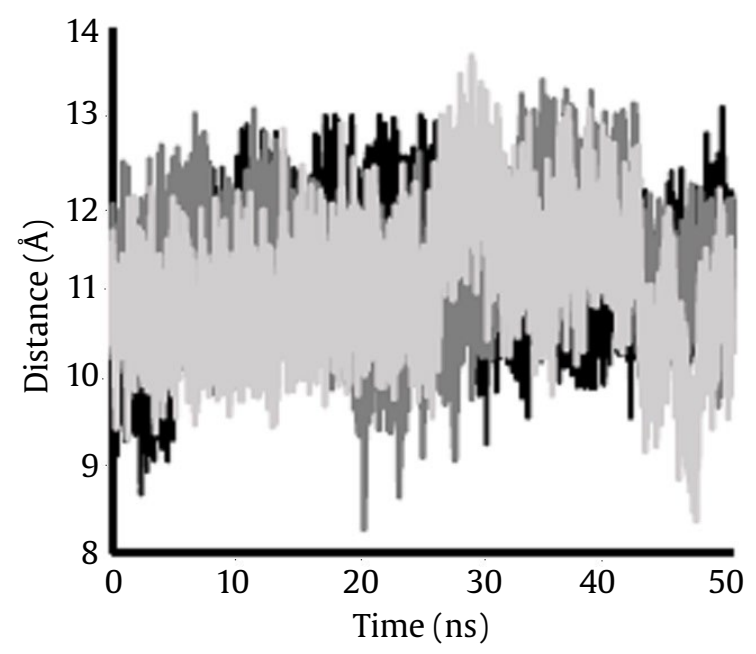

b

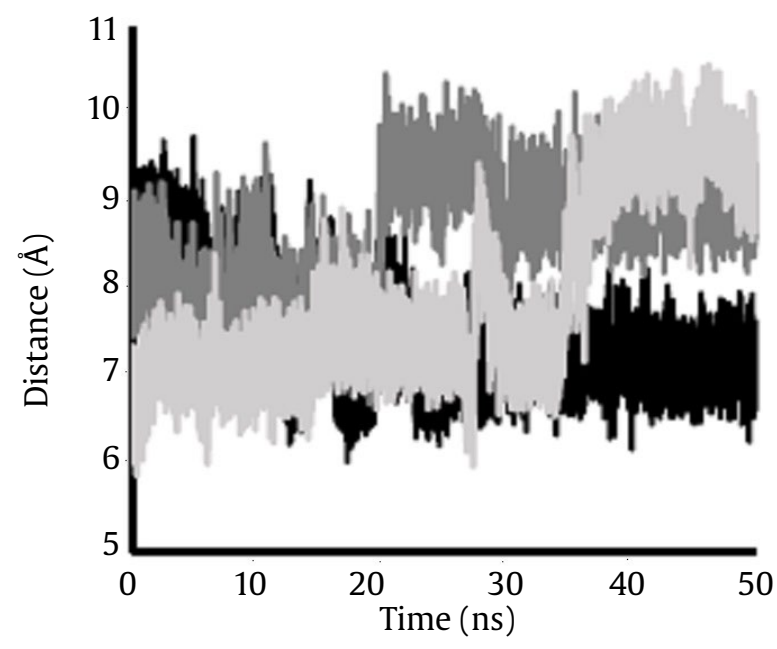

d
Run 1

Figure 4. (a) The electrostatic energy, and (b) the interaction distance of Lys20-Glu89 salt-bridge pair, while (c) the electrostatic energy, and (d) the interaction distance of Glu21-Arg142 salt-bridge pair 

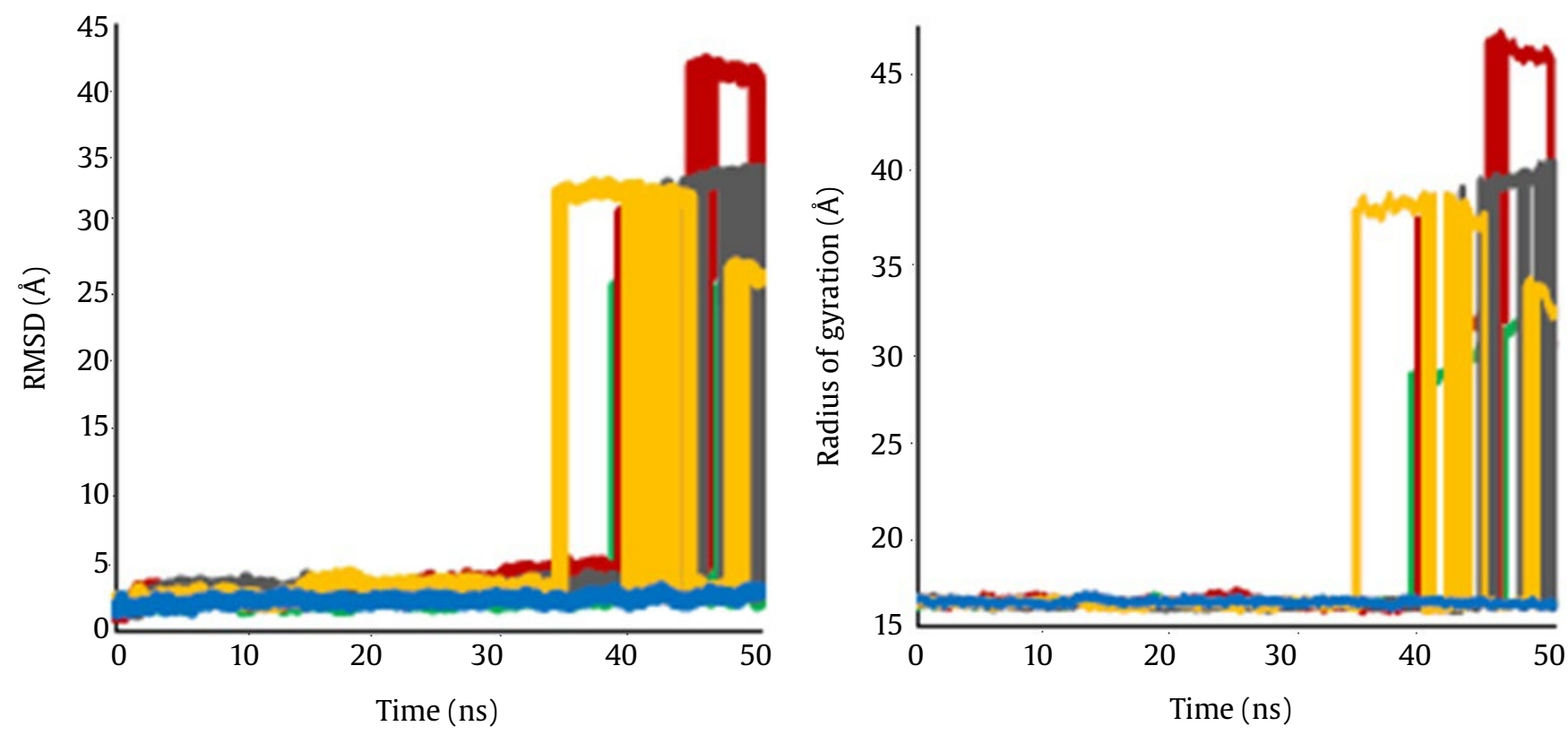

a

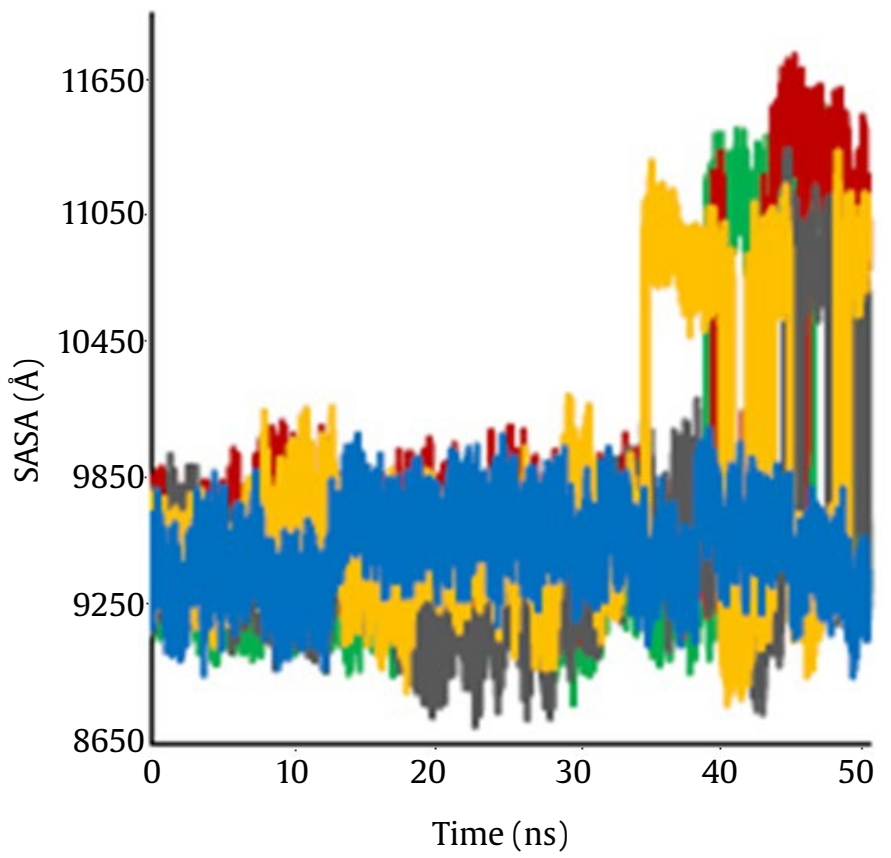

b

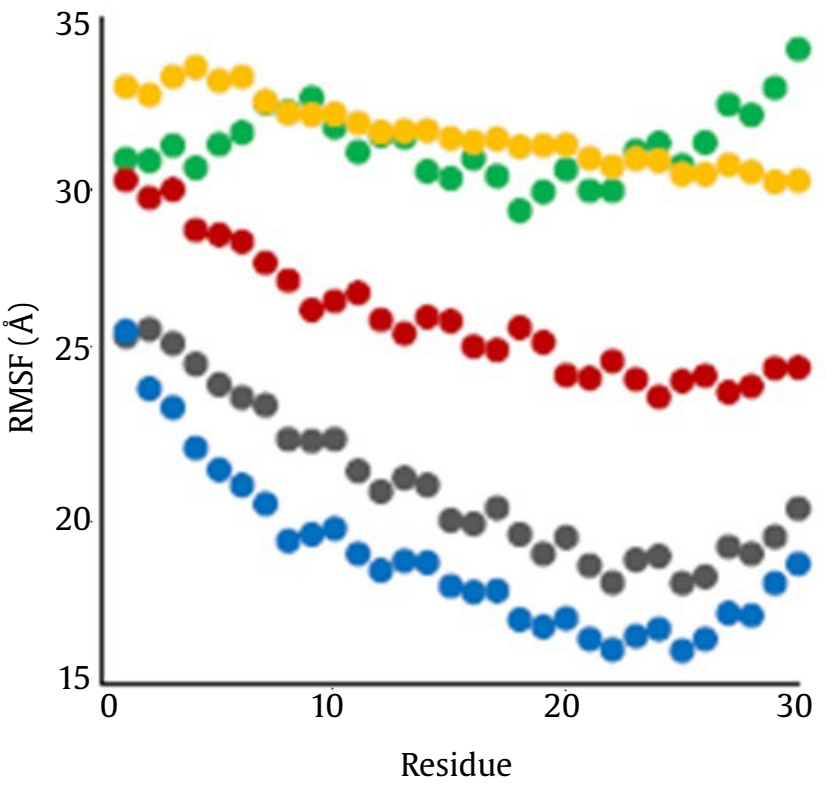

C

d

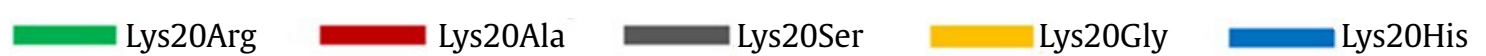

Figure 6. Stability analysis of GLP-1 mutants during 50 ns simulation based on (a) RMSD values, (b) SASA, (c) Radius of gyration, and (d) RMSF 
Wild-type
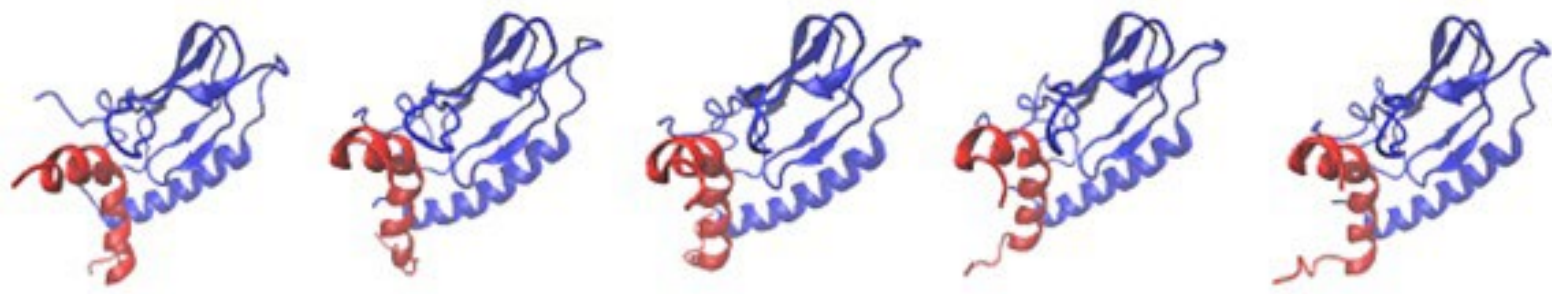

Lys20Arg
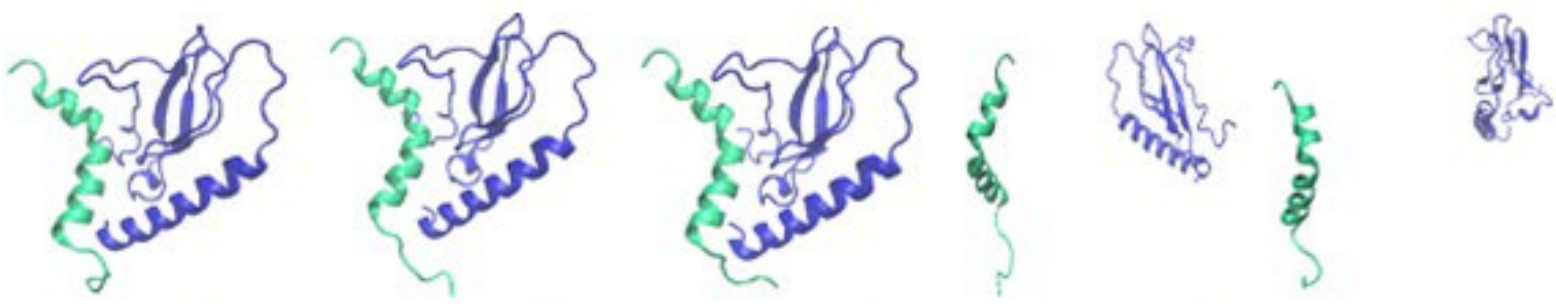

Lys20Ala
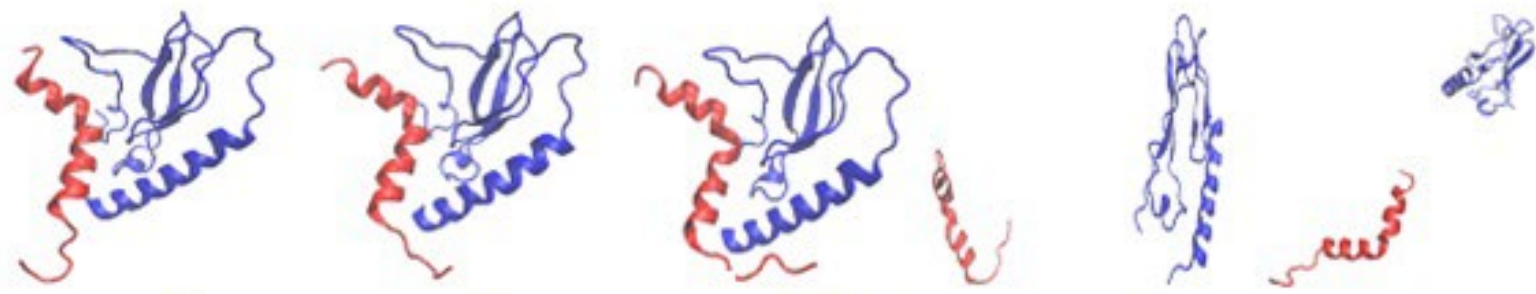

Lys20Ser
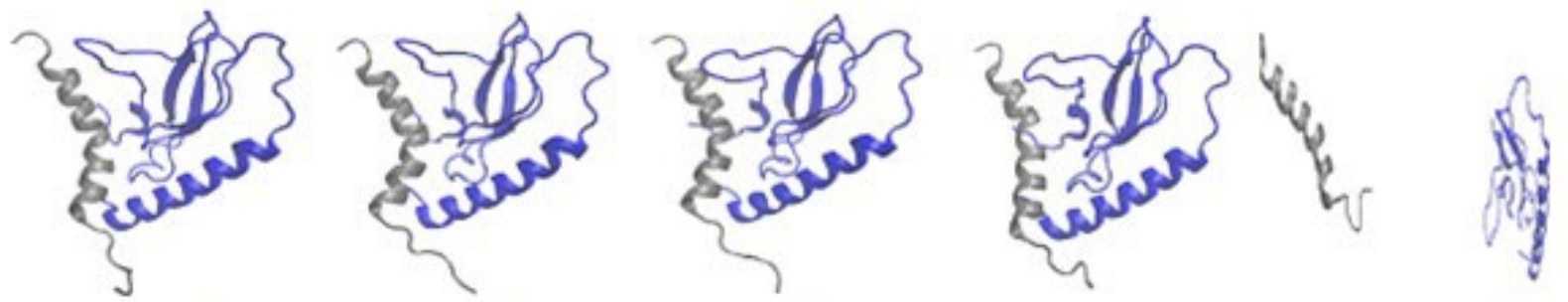

Lys20Gly
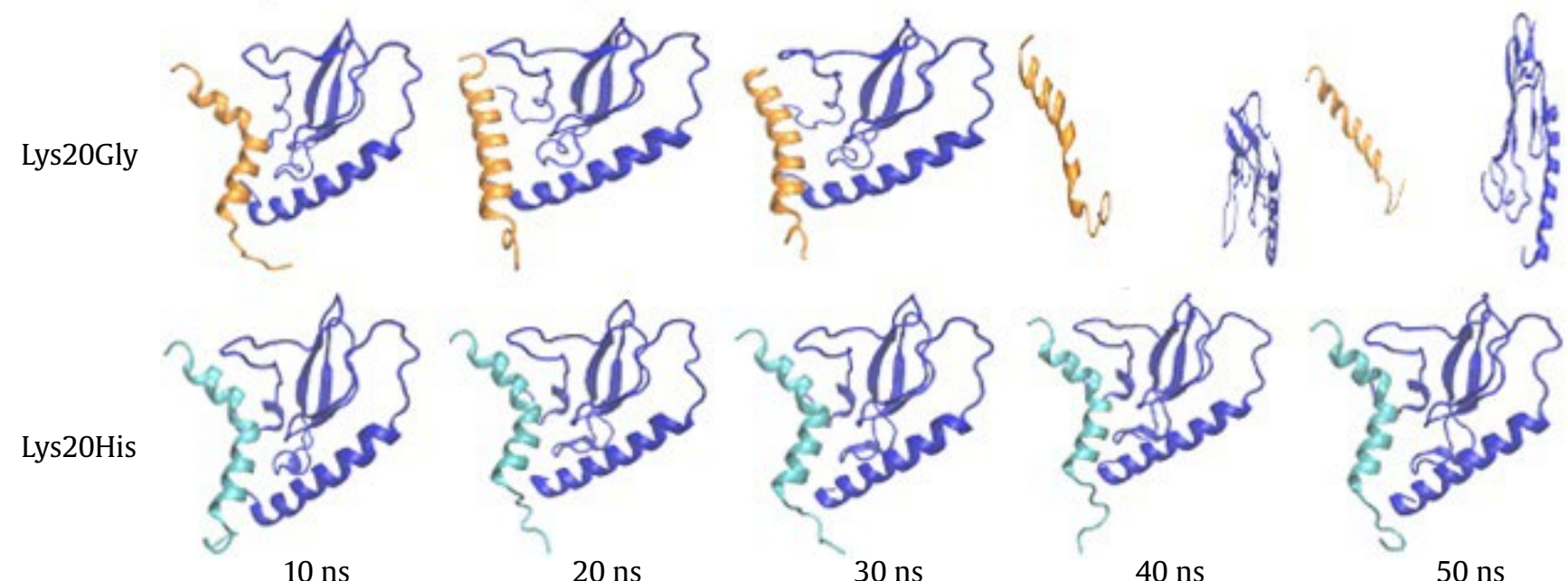

Figure 7. The binding evolution of Wild-type GLP-1, GLP-1 mutants, and GLP-1R receptor during the 50 ns simulation. Notice that Lys20His remains stable (docked) as compared to other mutants which detach from the receptor above $30 \mathrm{~ns}$. The dark blue color denotes the GLP-1R receptor 

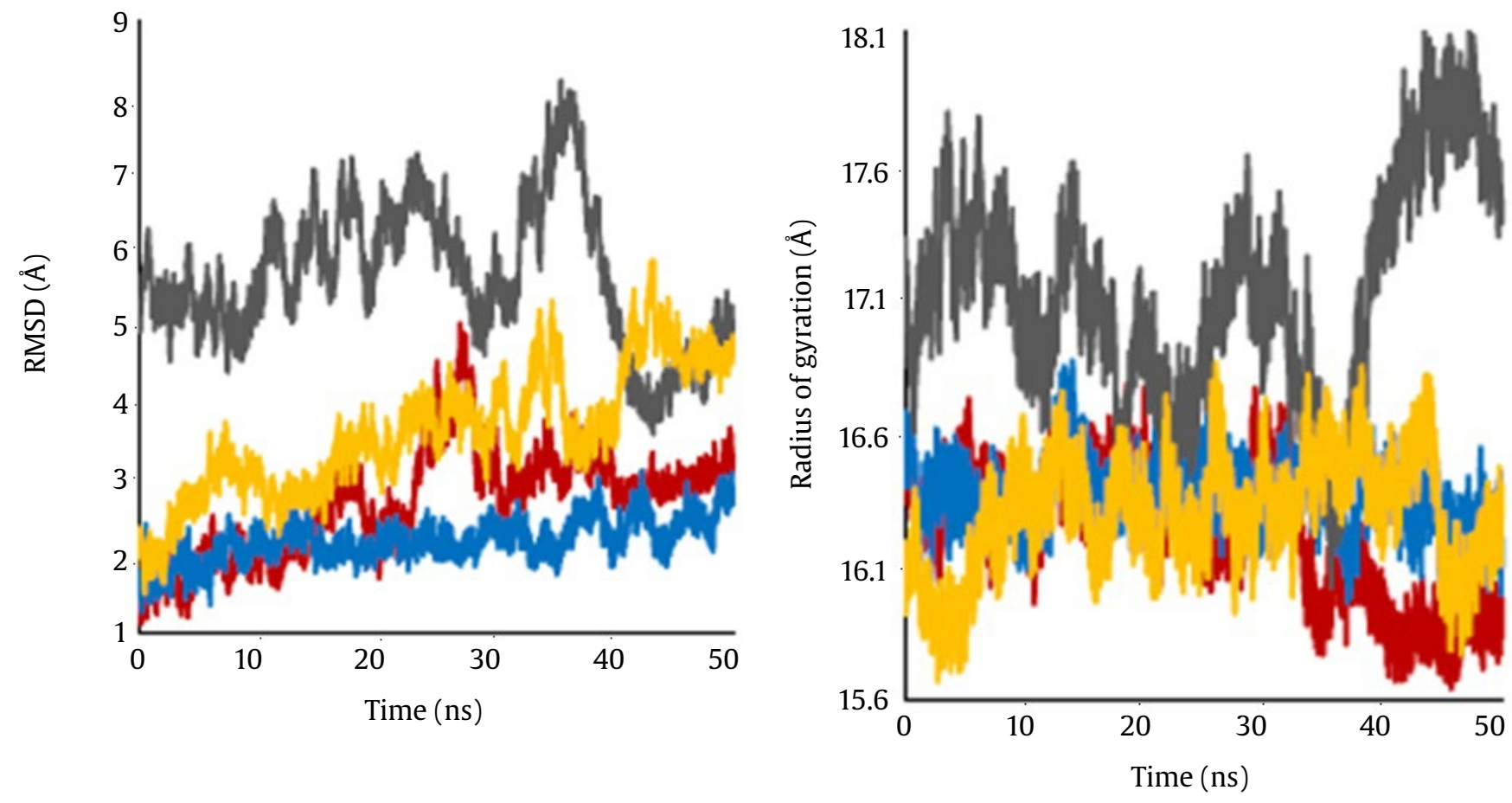

a
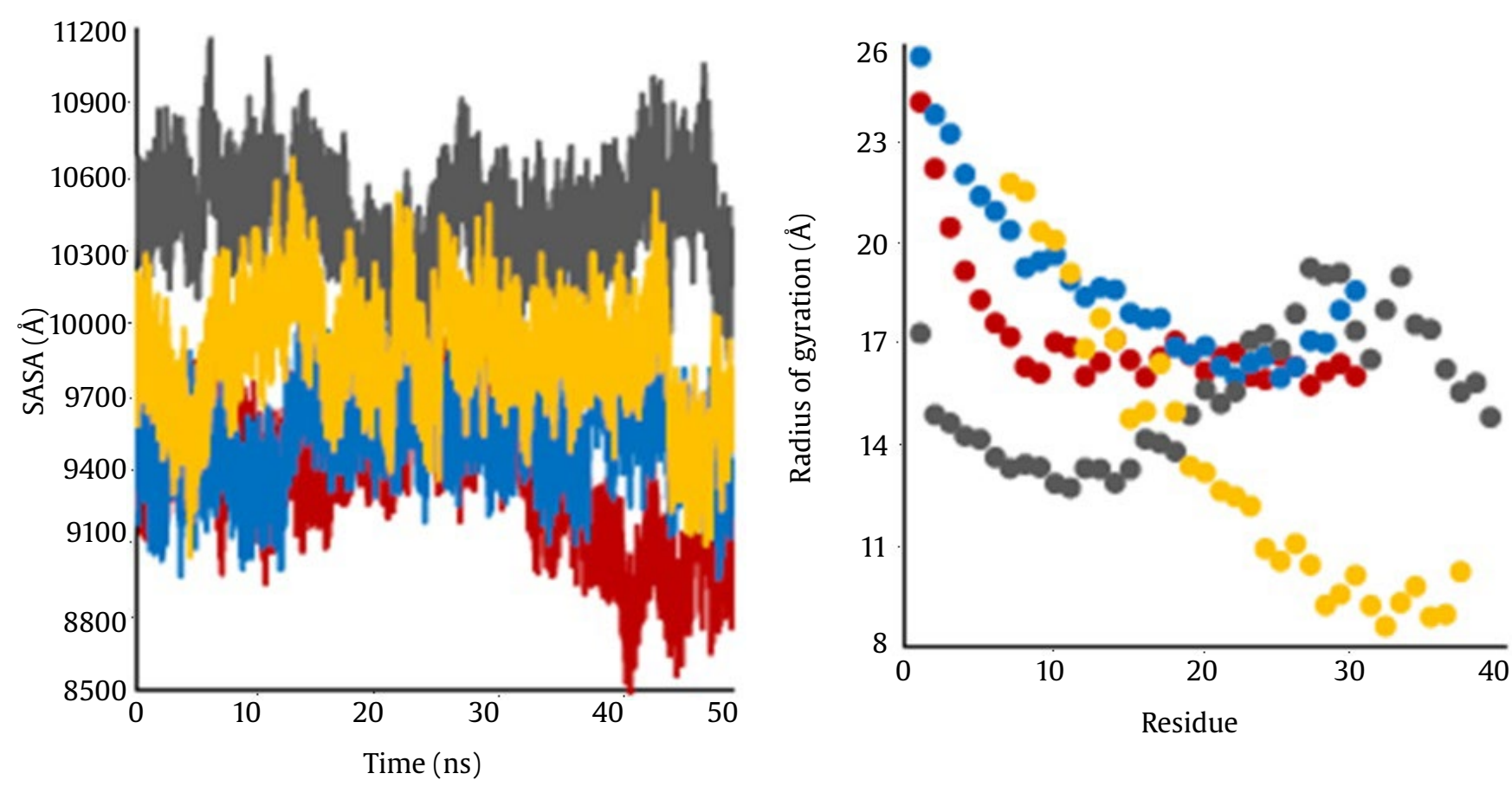

c

d

Wild-type Lys20His mutant

Exenantide Liraglutide

Figure 8. The stability analysis of mutant Lys20His as compared to the wild-type GLP-1, exenatide, and liraglutide during 50 ns molecular dynamics simulation 
Table 3. The binding affinity of GLP-1R receptor and the wild-type GLP-1, Lys20His mutant, exenatide, and liraglutide

\begin{tabular}{lllc}
\hline Protein & \multicolumn{2}{c}{$\Delta \mathrm{G}_{\text {bind }} \pm \mathrm{SD}(\mathrm{kcal} / \mathrm{mol})$} & $\begin{array}{c}\text { Number } \\
\text { of } \\
\text { Amino } \\
\text { Acid }\end{array}$ \\
\cline { 2 - 4 } & MMGBSA & MMPBSA & 30 \\
WT GLP-1 & $-91.3218 \pm 9.1883$ & $-91.105 \pm 10.5689$ & \\
Mutant & & & 30 \\
Lys20His & $-89.7866 \pm 7.5864$ & $-93.906 \pm 7.8009$ & 31 \\
Liraglutide & $-88.3018 \pm 6.8809$ & $-89.9955 \pm 7.7461$ & 39 \\
Exenatide & $-121.7081 \pm 6.9113$ & $-129.8063 \pm 7.8321$ & 39 \\
\hline
\end{tabular}

\section{Discussion}

The molecular dynamics simulation of the docked structure of the wild-type GLP-1 and the GLP-1R receptor was run three times for 50 ns each, and at $310 \mathrm{~K}$. The results have shown that those three runs of simulation did not produce a drastic conformational change (the docked structures are stable in each run). The stability of the docked structures is maintained during the simulation as demonstrated by the RMSD value of the backbone of protein-protein docking (Maiorov and Crippen 1994) which range from 2.47 to $6.1 \AA$.

The salt-bridge interactions between GLP-1 and GLP-1R binding were analyzed by identifying the positive and negative polar residue pairs that interact actively during the simulations (Winters and Day 2002). Salt-bridge interaction contributes essential roles in the flexibility and the stability of the protein structures, and also the balance of the docked structures of the protein-protein interaction (Horovitz et al. 1990; Xu et al. 1997; Karshikoff and Jelesarov 2008; Gribenko et al. 2009; Jelesarov and Karshikoff 2009). In Table 2, it was found that two active salt-bridges, Lys20-Glu89 and Glu21-Arg142, are found (existed) in each simulation and assumed as the essential salt-bridge pairs in maintaining the stability of the docked structures. These salt bridge pairs were further selected as the candidates for the point mutation. Recall that the salt-bridge pair Lys20Glu89 means the electrostatic interaction between Lys20 (in the GLP-1 ligand) and Glu89 (in the GLP-1R receptor). Since the mutation was designed for the ligand, so the candidate for mutation are Lys20 and Glu21 which further tested and selected based on the results of molecular dynamics simulation.

The electrostatic energy of Lys20-Glu89 and Glu21-Arg142 salt-bridge pairs during simulation were shown in Figure 4. The electrostatic interaction reinforces the non-covalent interaction in the protein structure, as emphasized by this Ref (Kumar and Nussinov 2002). Based on the electrostatic energy analysis, Glu21-Arg142 has stronger interaction as compared to the Lys20-Glu89 salt-bridge pair. The electrostatic energy of the Glu21-Arg142 pair has a more negative value $(-85.293 \pm 7.633 \mathrm{kcal} / \mathrm{mol})$ and shorter backbone distance $(8.005 \pm 0.948 \AA$.) as compared to Lys20-Glu89 which has less negative electrostatic energy $(-13.269 \pm 12.669 \mathrm{kcal} / \mathrm{mol})$ and a longer backbone distance $(11.157 \pm 0.688 \AA)$. The distance formed between two interacting residues influences the amount of electrostatic energy formed (Dominy et al. 2004; Maleki et al. 2013). The zero-value of electrostatic energy of the Lys20-Glu89 pair corresponds to a longer distance of separation between those two residues which exceeds the cutoff set up by the NAMD program (Ryckaert et al. 1977; Phillips et al. 2005).

From the electrostatic energy, the salt-bridge pair of Lys20-Glu89 was the weakest among other salt-bridge pairs in the binding of GLP-1 and GLP$1 \mathrm{R}$ complexes. Based on that result, we decided to make some point mutations of Lys20 residue to find the best replacement of that amino acid which could enhance the binding stability of the protein-protein docking. The Lys20 residue is a positive polar amino acid group, while its partner Glu89 is a negatively polar group. To analyze the effect of mutations on the binding stability, some residues must be selected from the same positive polar group such as Arginine and Histidine. The mutant candidates can also be chosen from the neutral polar group, such as Serine and Glycine, and nonpolar group like Alanine (Jonson and Petersen 2001; Wei et al. 2007). By using the popular convention for mutation symbol, the Lys20Arg means a point mutation by replacing Lysine with Arginine.

From the five designed mutants (Lys20Arg, Lys20Ala, Lys20Ser, Lys20Gly, Lys20His), Lys20His turned out to have the most negative $\Delta \Delta G_{\text {solv }}\left(\Delta \Delta G_{\text {solv }}\right.$ $=-5.135 \mathrm{kcal} / \mathrm{mol}$ ) as compared to other mutants $\left(\Delta \Delta G_{\text {solv }}=-0.5705 \mathrm{kcal} / \mathrm{mol}\right.$ for Lys20Ala, $\Delta \Delta G_{\text {solv }}$ $=+5.342 \mathrm{kcal} / \mathrm{mol}$ for Lys20Gly, $\Delta \Delta G_{\text {solv }}=+10.437$ $\mathrm{kcal} / \mathrm{mol}$ for Lys20Ser and $\Delta \Delta G_{\text {solv }}=+20.512 \mathrm{kcal} /$ mol for Lys20Arg). This implies that Lys20His is the best mutant candidate to be developed as a GLP-1R agonist. 
To evaluate the binding stability of GLP-1's mutants with GLP-1R, the molecular dynamics simulation of those five mutants (Lys20Arg, Lys20Ala, Lys20Ser, Lys20Gly, Lys20His) were performed at 310 $\mathrm{K}$ for 50 ns. In Figure 6, some molecular dynamics parameters such as RMSD, SASA (Solvent Accessible Surface Area indicates the area of protein's interior exposed by the solvent), Radius of gyration (the protein compactness), and RMSF (The Root Mean Square Fluctuation, or the flexibility of each residue) were shown as a function of simulation time. Based on the RMSD values, the binding of Lys20His mutant to its receptor was very stable as indicated by the minimum amount of RMSD which remained constant until the end of simulation at $50 \mathrm{~ns}$. On the other hand, the other four mutants began to detach from the receptors at 34.57 ns for Lys20Gly, at 38.98 ns for Lys20Arg, at 39.236 ns for Lys20Ala, and at 42.704 ns for Lys20Ser as seen in Figure 7. The same situation can be found in the value of the radius of gyration. The value of the radius of gyration and RMSD of Lys20His mutant remained stable during the simulation, while the other four mutants underwent a quite drastic change (See Figure $6 \mathrm{~b}$ ). The SASA value of Lys20His mutant has a stable value ranging from 8952 to $10073 \AA 2$ (1121 Å2 area change), whereas the SASA value of other mutants has a more significant range and area change. For the Lys20Arg the SASA value ranging from 8829 to $11607 \AA 2$ (2778 2 area change), Lys20Ala ranging from 9050 to 11767 Å2 (2717 Å2 area change), Lys20Ser ranging from 8730 to $11337 \AA 2$ (2607 $\AA 2$ area change) as seen in Figure $6 c$. The enlargement of the SASA area directly corresponds to the breaking of the salt-bridge pairs which in this case displayed the detachment process of the ligand from the receptor (Durham et al. 2009). The RMSF analysis showed that the Lys20His mutant has the most stable structures as compared to other mutants as demonstrated by the lower RMSF values (See Figure 6d).

Figure 8 showed the docking (binding) stability of Lys20His GLP-1 mutant to GLP-1R as compared to the wild-type GLP-1 and the available peptide agonist drugs in the market, exenatide, and liraglutide during the 50 ns simulation. As shown in Figure 8, the Lys20His mutant showed good results (more stable binding) based on its lower RMSD, Radius of gyration and SASA values. The RMSF analysis also indicated that the replacement of Lysine- 20 with Histidine maintained the compactness of the docked structures.
The SASA value of Lys20His mutant was ranging from 8951 to $10073 \AA^{2}$ which was almost similar to the value of the wild-type that range from 8479 to 10200 $\AA^{2}$. Comparing to other mutants, the replacement of Lysine-20 with Histidine did not change the SASA's value too much and kept the docked structure in a stable state. The SASA value of Lys20His mutant was also lower than exenatide (9712-11151 $\AA^{2}$ ) and liraglutide (9040-10665 $\left.\AA^{2}\right)$. Overall based on those molecular dynamics parameters, we can assume that our designed mutant Lys20His has better binding stability as compared to other mutants and the available peptide agonist drugs on the market.

To evaluate the binding affinity of Lys20His mutant, GLP-1 wild-type, exenatide, and liraglutide to the GLP-1R receptor, the binding energy calculation using the MM/PBSA and MM/GBSA methods were performed. The results of those methods were presented in Table 3. The binding affinity calculation was intended to describe and analyze the strength of protein-protein interaction (Hou et al. 2011; Beard et al. 2013). Based on the data in Table 3, the Lys20His mutant has stronger binding energy as compared to liraglutide. When Lys20His mutant was compared to wild-type GLP-1, it has a smaller standard deviation, both in MM/PBSA or MM/GBSA method. Those results suggest that replacing Lysine-20 with Histidine improves the docking stability of GLP1 with the GLP-1R receptor. The slight difference between the binding affinity of the Lys20His mutant with the wild-type GLP-1 and liraglutide reflects the similar energetic performance of this mutant as compared to the natural agent. The stronger binding affinity of exenatide as compared to Lys20His was expected due to a bigger size and more residues (39 residues in exenatide) involved in the proteinprotein interactions since the number of amino acids as we know significantly affects the calculation of the binding energy (Genheden and Ryde 2015; Duan et al. 2016).

The replacement of Lysine-20 with Histidine has increased the binding stability as compared to other mutants and analogs as shown by its structural parameters (Figure 6) and energetical parameters (Table 3). The replacement of Lysine-20 with Histidine also creates a stronger and flexible binding due to the nature of Histidine which can be in the interior or exterior of the protein and more comfortable to move proton (change from neutral to positively charged residue) as compared to Lysine 
which prefers on the outside of protein and interact with other carboxy groups, anions or involved in saltbridge interaction. There are some efforts to increase the binding quality of GLP- 1 and GLP-1R receptor, including these references (Green et al. 2003; Green et al. 2004; Dods and Donnelly 2016). According to (Dods and Donnelly 2016) who performed mutation on GLP-1R, the ligand-receptor binding mostly occurred between the $\mathrm{N}$ terminal domain (NTD) of GLP-1 peptides such as His1, Glu3, Ser 8, Asp9 and the cavity (pocket interaction) of GLP-1R, with a minority of GLP-1R amino acids interacts with the helical section of the GLP-1 (from Ala18 to Val27). Most of the effort in modifying GLP-1 have been focused on NTD to avoid the rapid destabilization of GLP-1 by dipeptidyl peptidase IV (DPP IV) enzyme, for example (Green et al. 2003) who performed mutation of Glu9 (in our GLP-1 structure, Glu3) with Proline, Phenyl, and Tyrosine. In this paper, we have demonstrated that the protein modification can be done in other section of GLP-1 by replacing Lys20 with His20 and still produces better binding stability as compared to the wild-type of GLP-1.

\section{Conclusion}

We have conducted a computational approach of protein-protein docking, molecular dynamics simulation, free energy perturbation, and molecular mechanics binding affinity calculation to design a stable GLP-1 ligand that has the strongest binding affinity to the GLP-1R receptor. Out of five mutant candidates (Lys20Arg, Lys20Ala, Lys20Ser, Lys20Gly, Lys20His), Lys20His has shown good interaction stability with GLP-1R receptor as indicated by some molecular dynamics parameters of RMSD, RMSF, the radius of gyration and SASA. The binding affinity calculation using MM/PBSA and MM/GBSA technique has also shown a strong binding affinity of Lys20His with the GLP-1R receptor. In summary, we have successfully designed a new mutant of GLP-1 incretin hormone which showed almost similar stability structurally and energetically to the wild-type GLP1 and a better balance as compared to the peptide agonist drugs available in the market, exenatide, and liraglutide.

\section{Acknowledgements}

The authors would like to express their gratitude to The Ministry of Research Technology and Higher Education of The Republic of Indonesia for funding this research through Grant No. 079/SP2H/IT/DRPM/ II/2016.

\section{References}

Ahrén B, Ahren B. 2011. GLP-1 for type 2 diabetes. Experimental Cell Research 317:1239-1245. DOI:10.1016/j. yexcr.2011.01.010

American Diabetes Association. 2012. Diagnosis and classification of diabetes mellitus. Diabetes Care 35:64-71. DOI:10.2337/dc12-s064

Anderberg RH et al. 2016. GLP-1 is both anxiogenic and antidepressant; divergent effects of acute and chronic GLP-1 on emotionality. Psychoneuroendocrinology 65:54-66. DOI:10.1016/j.psyneuen.2015.11.021

Baggio LL, Drucker DJ. 2007. Biology of incretins: GLP-1 and GIP. Gastroenterology 132:2131-2157. DOI:10.1053/j. gastro.2007.03.054

Bai X et al. 2016. A new GLP-1 analogue with prolonged glucose-lowering activity in vivo via backbone-based modification at the N-terminus. Bioorganic and Medicinal Chemistry 24:1163-1170. DOI:10.1016/j. bmc.2016.01.036

Beard $\mathrm{H}$ et al. 2013. Applying physics-based scoring to calculate free energies of binding for single amino acid mutations in protein-protein complexes. PLOS ONE 8:1-11. DOI:10.1371/journal.pone.0082849

Briones M, Bajaj M. 2006. Exenatide: A GLP-1 receptor agonist as novel therapy for Type 2 diabetes mellitus. Expert Opin Pharmacother 7:1055-1064.

Chang X et al. 2001. Structure and folding of glucagon-like peptide-1-(7-36)-amide in aqueous trifluoroethanol studied by NMR spectroscopy. Magnetic Resonance in Chemistry 39:477-483. DOI:10.1002/mrc.880

Chen R et al. 2003. ZDOCK: An initial-stage protein-docking algorithm. Proteins: Structure, Function and Genetics 52:80-87. DOI:10.1002/prot.10389

De Maturana RL et al. 2003. The isolated N-terminal domain of the glucagon-like peptide-1 (GLP-1) receptor binds exendin peptides with much higher affinity than GLP1. Journal of Biological Chemistry 278:10195-10200. DOI:10.1074/jbc.M212147200

Deller MC et al. 2016. Protein stability: a crystallographer's perspective. Acta Crystallogr F Struct Biol Commun 72:72-95. DOI:10.1107/S2053230X15024619

Dods RL, Donnelly D. 2016. The peptide agonist-binding site of the glucagon-like peptide-1 (GLP-1) receptor based on site-directed mutagenesis and knowledge-based modelling. Bioscience Reports 36:285. DOI:10.1042/ BSR20150253

Dominy BN et al. 2004. An electrostatic basis for the stability of thermophilic proteins. Proteins: Structure, Function and Genetics 57:128-141. DOI:10.1002/prot.20190

Doyle ME, Egan JM. 2007. Mechanisms of action of glucagonlike peptide 1 in the pancreas. Pharmacol Ther 113:546-593. DOI:10.1016/j.pharmthera.2006.11.007 
Drucker DJ. 2001. Development of glucagon-like peptide-1based pharmaceuticals as therapeutic agents for the treatment of diabetes. Current Pharmaceutical Design 7:1399-1412. DOI:10.2174/1381612013397401

Duan LL et al. 2016. Large-scale molecular dynamics simulation: effect of polarization on thrombin-ligand binding energy. Scientific Reports 6:1-11. DOI:10.1038/ srep31488

Durham E et al. 2009. Solvent accessible surface area approximations for rapid and accurate protein structure prediction. Journal of Molecular Modeling 15:1093-1108. DOI:10.1007/s00894-009-0454-9

Genheden S, Ryde U. 2015. The MM/PBSA and MM/GBSA methods to estimate ligand-binding affinities. Expert Opinion on Drug Discovery 10:449-461. DOI:10.1517| 17460441.2015 .1032936

Green BD et al. 2003. Metabolic stability, receptor binding, cAMP generation, insulin secretion and antihyperglycaemic activity of novel N-terminal Glu9substituted analogues of glucagon-like peptide- 1 . Biological Chemistry 384:1543-1551. DOI:10.1515/ BC.2003.171

Green BD et al. 2004. Structurally modified analogues of glucagon-like peptide-1 (GLP-1) and glucosedependent insulinotropic polypeptide (GIP) as future antidiabetic agents. Current Pharmaceutical Design 10:3651-3662. DOI:10.2174/1381612043382774

Gribenko AV et al. 2009. Rational stabilization of enzymes by computational redesign of surface chargecharge interactions. Proceedings of the National Academy of Sciences 106:2601-2606. DOI:10.1073/ pnas.0808220106

Horovitz A et al. 1990. Strength and co-operativity of contributions of surface salt bridges to protein stability. Journal of Molecular Biology 216:1031-1044. DOI:10.1016/S0022-2836(99)80018-7

Hou T et al. 2011. Assessing the Performance of the MM/ PBSA and MM/GBSA Methods. I. The accuracy of binding free Energy calculations based on molecular dynamics simulations. J Chem Inf Model 51:69-82. DOI: $10.1021 /$ ci100275a

Humphrey W et al. 1996. VMD: Visual molecular dynamics. Journal of Molecular Graphics 14:33-38. DOI:10.1016/0263-7855(96)00018-5

Jelesarov I, Karshikoff A. 2009. Defining the role of salt bridges in protein stability. Protein Structure, Stability and Interactions 490:227-260. DOI:10.1007/978-159745-367-7

Jonson PH, Petersen SB. 2001. A critical view on conservative mutations. Protein Engineering 14:397-402. DOI:10.1093/protein/14.6.397

Karshikoff A,Jelesarov I. 2008. Salt bridges and conformational flexibility: effect on protein stability. Biotechnology and Biotechnological Equipment 22:606-611. DOI:10. $1080 / 13102818.2008 .10817520$

Kumar S, Nussinov R. 2002. Close-range electrostatic interactions in proteins. Chembiochem 3:604-617. DOI:10.1002/1439-7633(20020703)3:7<604::AIDCBIC604>3.0.CO;2-X

Li Y et al. 2010. Enhancing the GLP-1 receptor signaling pathway leads to proliferation and neuroprotection in human neuroblastoma cells. Journal of Neurochemistry 113:1621-1631.DOI:10.1111/j.1471-4159.2010.06731.x

Maiorov VN, Crippen GM. 1994. Significance of root-meansquare deviation in comparing three-dimensional structures of globular proteins. Journal of Molecular Biology 235:625-634. DOI:10.1006/jmbi.1994.1017
Maleki M et al. 2013. The role of electrostatic energy in prediction of obligate protein-protein interactions. Proteome Science 11:1-12 DOI:10.1186/1477-595611-S1-S11

McClean PL et al. 2011. The diabetes drug liraglutide prevents degenerative processes in a mouse model of alzheimer's disease. Journal of Neuroscience 31:65876594. DOI:10.1523/JNEUROSCI.0529-11.2011

Meloni AR et al. 2012. GLP-1 receptor activated insulin secretion from pancreatic $\beta$-cells: mechanism and glucose dependence. Diabetes, Obesity and Metabolism 9999:1-13. DOI:10.1111/j.1463-1326.2012.01663.x

Mostafa AM et al. 2015. Glucagon-like peptide 1(GLP-1)-based therapy upregulates LXR-ABCA1/ABCG1 cascade in adipocytes. Biochemical and Biophysical Research Communications 468:900-905. DOI:10.1016/j. bbrc.2015.11.054

Neidigh JW et al. 2001. Exendin-4 and glucagon-likepeptide-1: NMR structural comparisons in the solution and micelle-associated states. Biochemistry 40:13188-13200. DOI:10.1021/bi010902s

Olokoba $A B$ et al. 2012. Type 2 diabetes mellitus: a review of current trends. Oman Medical Journal 27: 269-273. DOI:10.5001/omj.2012.68

Phillips JC et al. 2005. Scalable molecular dynamics with। ppercase \{NAMD\}. J Comput Chem 26:1781-1802. DOI:10.1002/jcc.20289

Pierce BG et al. 2014. ZDOCK server: interactive docking prediction of protein-protein complexes and symmetric multimers. Bioinformatics 30:1771-1773. DOI:10.1093/bioinformatics/btu097

Pikkemaat MG et al. 2002. Molecular dynamics simulations as a tool for improving protein stability. Protein Engineering 15:185-192.DOI:10.1093/protein/15.3.185

Quoyer J et al. 2010. GLP-1 mediates antiapoptotic effect by phosphorylating bad through a $\beta$-arrestin 1 -mediated ERK1/2 activation in pancreatic $\beta$-cells. Journal of Biological Chemistry 285:1989-2002. DOI:10.1074/ jbc.M109.067207

Runge S et al. 2008. Crystal structure of the ligand-bound glucagon-like peptide-1 receptor extracellular domain. J Biol Chem 283:11340-11347. DOI:10.1074/ jbc.M708740200

Russell-Jones D. 2009. Molecular, pharmacological and clinical aspects of liraglutide, a once-daily human GLP-1 analogue. Molecular and Cellular Endocrinology 297:137-140. DOI:10.1016/j.mce.2008.11.018

Ryckaert JP et al. 1977. Numerical integration of the cartesian equations of motion of a system with constraints: molecular dynamics of n-alkanes. Journal of Computational Physics 23:327-341.DOI:10.1016/00219991(77)90098-5

Worldwide PDB. 2014. PDB Entry-4APD. Available at: https:// www.wwpdb.org/pdb?id=pdb_00004apd[Dateaccessed: 20 July 2016]

Henin J et al. 2017. In silico alchemy: A tutorial for alchmical free-energy perturbation calculation with NAMD. 2017. Available at: https://www.ks.uiuc.edu/Training Tutorials/namd/FEP/tutorial-FEP.pdf [Date accessed: 20 Oktober 2017]

Vilsbøll T et al. 2008. Liraglutide, a once-daily human GLP1 analogue, improves pancreatic B-cell function and arginine-stimulated insulin secretion during hyperglycaemia in patients with Type 2 diabetes mellitus. Diabetic Medicine 25:152-156. DOI:10.1111/ j.1464-5491.2007.02333.x 
Wei Y et al. 2007. NMR study and molecular dynamics simulations of optimized beta-hairpin fragments of protein G. Proteins 69:285-296. DOI:10.1002/ prot. 21494

Weyer C et al. 1999. The natural history of insulin secretory dysfunction and insulin resistance in the pathogenesis of type 2 diabetes mellitus. The Journal of Clinical Investigation 104:787-794. DOI:10.1172/jci7231
Winters MS, Day RA. 2002. Identification of amino acid residues participating in intermolecular salt bridges between self-associating proteins. Analytical Biochemistry 309:48-59. DOI:10.1016/S00032697(02)00296-8

Xu D et al. 1997. Hydrogen bonds and salt bridges across protein-protein interfaces. Protein Engineering 10:999-1012. DOI:10.1093/PROTEIN/10.9.999 